\title{
Communicating Wave Energy: An Active Learning Experience For Students
}

Trongnghia Huynh, Old Dominion University, USA

Gene Hou, Old Dominion University, USA

Jin Wang, University of Tennessee, USA

\begin{abstract}
We have conducted an education project to communicate the wave energy concept to high school students. A virtual reality system that combines both hardware and software is developed in this project to simulate the buoy-wave interaction. This first-of-its-kind wave energy unit is portable and physics-based, allowing students to conduct a number of hands-on activities. This system is the core component of an educational experience that integrates demonstration and hands-on learning, with an aim of introducing the wave energy conversion process to students in an interactive environment. Presentations have been made at two different high schools with diverse student populations, and students involved in this project rated very positively about their learning experience. As revealed by their feedback, the virtual environment and its combination with the hardware are the most important factors that help students to appreciate the knowledge in the wave energy conversion process.
\end{abstract}

Keywords: Wave Energy; Virtual Reality System; Demonstration; Hands-On Learning

\section{INTRODUCTION}

6 ur project is concerned with educating younger students for knowledge and awareness in wave energy. Ocean waves, generated by wind blowing over the water surface, have tremendous energy which can be captured and converted into electricity. With the rising demand for energy, growing consumption of oil and gas, and increasing global warming, waves offer an attractive green and renewable energy source and have generated considerable interest in research, development and test in recent years. The US coast is one of the few places in the world that have rich potential for wave energy (other areas include Canada, northwestern Europe, Southern Africa and Australia). However, currently the exploration of wave energy is still at an early stage, and commercial wave power harnessing systems are merely in their prototype testing phase, due to several technological challenges in the design of robust and cost-effective devices (Falnes, 2002; Cruz, 2010; McCormic, 2007). It is our hope that by introducing the wave energy concept to the younger generation, particularly the high school students, and teaching them the basic knowledge, we can provide early exposure to the young minds in wave energy and other renewable energy, promote research and development in the field, and, in the long run, prepare a knowledgeable and responsible workforce that can contribute to the scientific and technological advances in renewable energy and related science and engineering.

To achieve this goal, we have carried out the project in a way that can effectively stimulate students' interest and promote their learning. There are three commonly used methods, among others, for teaching and learning (Westwood, 2008; Renk1, Atkinson, Maier, \& Staley, 2002; Kyriacou, 1992). Verbal instruction, or lecturing, is perhaps the most ubiquitous way of teaching, as typically seen when a teacher gives a lecture to students sitting in a classroom. The disadvantage of verbal instruction (alone) is that it often lacks active learner involvement, thus frequently viewed (and criticized) as being passive learning for students. The second method is learning by demonstration, where a teacher simultaneously explains the concepts and demonstrate what is explained, typically through visual or auditory means. The third method is hands-on learning, which actively involves students in manipulating physical objects in order to gain knowledge or understanding. There have been many educational studies performed on the analysis and comparison of these methods. In particular, several results (Hearns, Miller, \& Nelson, 2010; Kontra, Lyons, Fischer, 
\& Beilock, 2015; Hillman, 2011) have indicated that when hands-on experience is incorporated, the learning is more effective than using demonstration only, when measuring long-term memory and recall of knowledge. Thus, throughout this project, we have made special efforts to integrate these different approaches, particularly the demonstration and hands-on learning, in our wave energy education. In this way, we are promoting experiential learning, or "learning through reflection on doing" (Beard, 2010; Itin, 1999), with an aim or providing an active learning experience for students.

An essential component of this education project is a wave energy demonstration unit. Our design of the demonstration unit is based on the concept of virtual reality (VR) (Huynh, Hou, Wang, Hou, \& Kotinis, 2015), defined as "a mosaic of technologies that support the creation of synthetic, highly interactive three- dimensional spatial environments that represent real or non-real situations" (Mikropoulos \& Natsis, 2011). VR has been incorporated by many educational institutions in science and engineering classroom teaching, such as factory production process, chemistry, electrical and mechanical engineering laboratories (Gadre, Cudney, \& Crons, 2011; Ong, Mannan, 2004; Dalgarno, Bishop, Adlong, \&Bedgood, 2009; Saleh, Mohamed, \& Madkour, 2009; Goeser, Johnson, Hamza-Lup \& Schaefer, 2011). Recent advances in software-hardware interfacing have increased the level of sophistication and broadened the range of applications for VR that allows the virtual environment to interact with physical models in real time (Hu, \& Zeigler, 2005; Richardson, Adamo-Villani, Carpenter, \& Moore, 2006; Morris, Swewll, Blevins, Barbagli, \& Salsbury, 2004; Fu, Chen, Cai, 2011; Crosier, Cobb, \& Wilson, 2002).

Using the virtual reality concept, we have designed and developed a first-of-its-kind wave energy demonstration unit with hardware-software integration. The system is portable and physics-based, allowing students to conduct a number of hands-on activities and promoting their interest. We have made the presentations at two public high schools with diverse student populations: a vocational school in New Jersey (Camden County Technical School, or CCTS), and a highly-ranked school in Virginia (Princess Anne High School, or PAHS). A short survey is conducted after the presentation for all involved students at each school.

The remainder of the paper is organized as follows. We first provide the details of our wave energy virtual reality system, and the set-up for our demonstration. Next, we describe the format of school presentations that combine the lecturing, demonstration, and hands-on learning. We then highlight the experience of our visits and presentations to students at CCTS and PAHS, as well as the results of student survey. Finally, we conclude the paper with some discussion.

Figure 1. Hardware Set-up

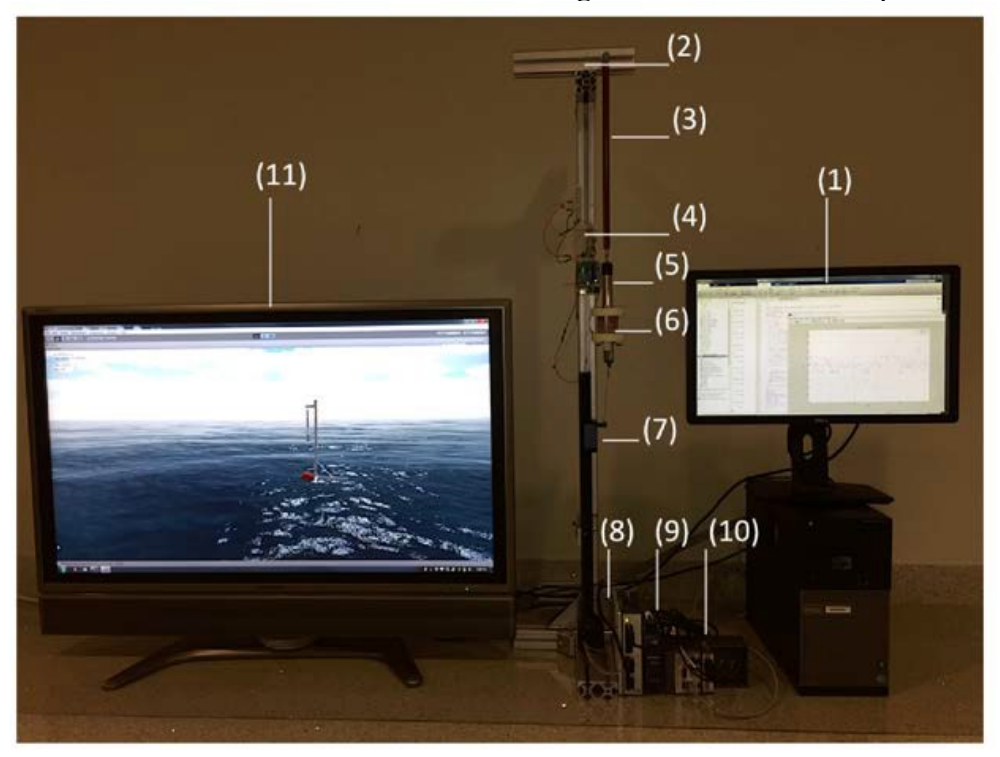

(1) - MATLAB Simulator

(2) - Crane Mechanism

(3) - Linear Spring

(4) - Amplified LED Circuit

(5) - Permanent Magnet

(6) - Copper Solenoid

(7) - Stepper Motor \& Rail

(8) - Stepper Amplifier

(9) - DC Power Supply

(10)- C-Rio Controller

(11) - Unity Visual Engine 
Figure 2. Data Flow Diagram

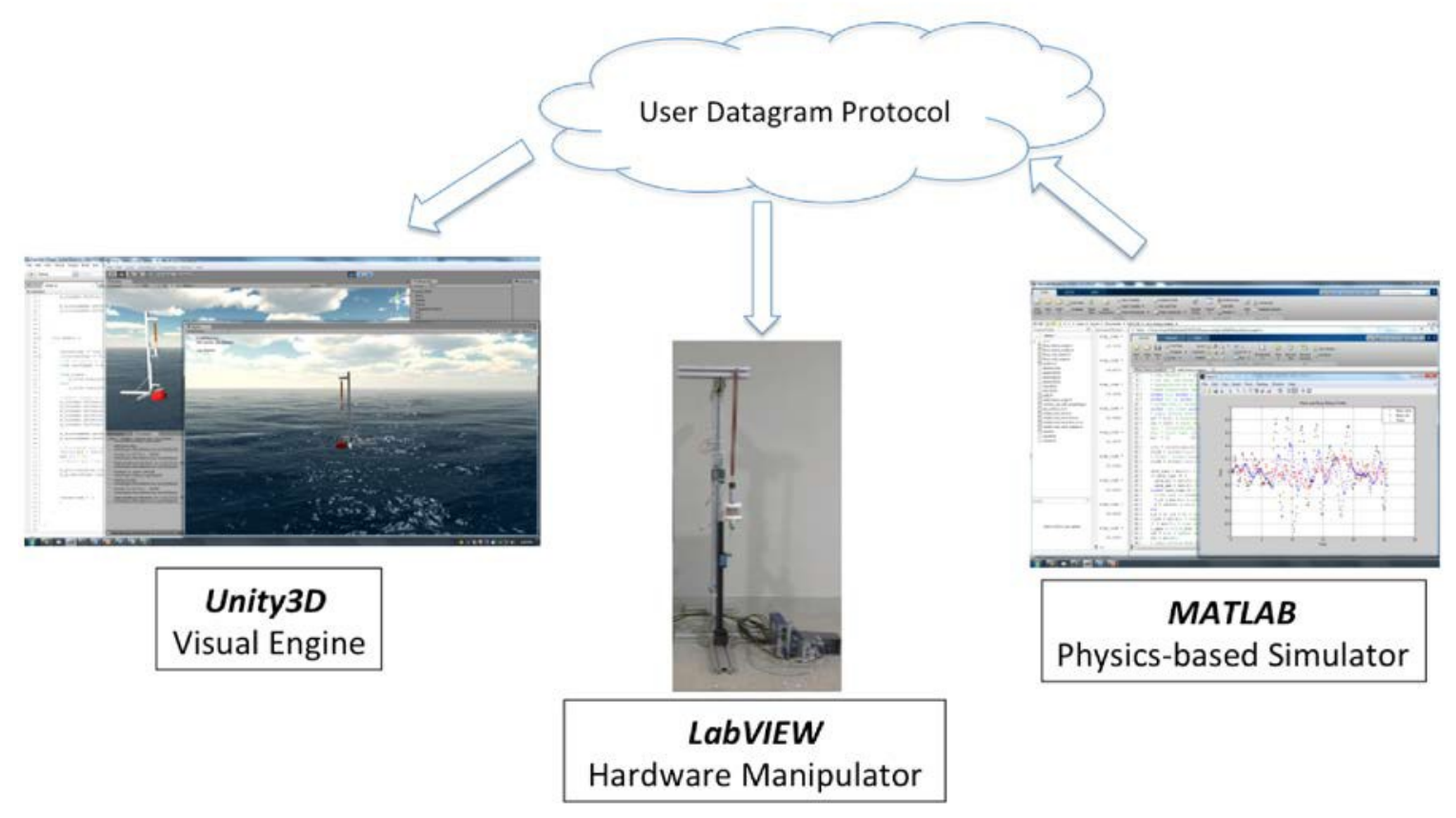

II. WAVE ENERGY VIRTUAL REALITY SYSTEM

The wave energy convertor (WEC) machine developed in our project is a hardware in-the-loop virtual reality system. It was designed as an interactive and visual enhanced module to demonstrate the idea on how to convert the mechanical wave energy into electrical current. The WEC hardware system as a whole is presented in Fig. 1. The whole system is made of three parts; the MATLAB simulator, the crane mechanism, and the Unity visual engine. The data flow between them is shown in Fig. 2. The MATLAB simulator marked by (1) in Fig. 1 simulates the wave-buoyance interaction numerically, generating two sets of simulation results. One is the wave motion under the given sea state and wind speed, and the other is the motion profile of the buoy. Both the motion profiles of the buoy and the waves will be transmitted wirelessly to the Unity visual engine to create a scene of buoy-wave interaction in a virtual environment. The buoy motion profile will be transmitted wirelessly to the crane mechanism to physically move the magnetic rod. The crane mechanism is marked by (2) in Fig. 1, the magnetic rod by (5), and the Unity visual engine by (11).

The crane mechanism comprises of a crane, a magnetic rod, a coil, an LED board, and a linear spring. The crane, which is about 40 inches in height, was made to offer the mounting places and hold the various parts in place. While the 6-inch-long magnetic rod goes up and down synchronously with the buoy motion, the copper solenoid with18gauge diameter stays motionless. This coil converts the change in flux created by the moving magnetic rod to the electrical current so as to power the LED on the circuit board. The linear spring anchored at the highest point of the crane holds the magnetic rod in place and allows it to move only in the desired vertical direction. Once the motion profile of the buoy is received, the LabVIEW along with a C-Rio controller will move the magnetic rod accordingly. Thus, the magnetic rod is a hardware simulating the buoy in this wave energy conversion machine.

The MATLAB physics-based simulator acts as the brain of the whole project while the User Datagram Protocol, UDP, is utilized to transmit the buoy motion profile in every time interval one at a time to the other two blocks: the LabVIEW and crane mechanism to incite the motor and the Unity $3 \mathrm{D}$ visual engine to envision the simulation. The UDP is the networking protocol that allows the each module direct access to the data transport service. It allows the simulation information generated through MATLAB be transmitted in a lock step synchronously to both Unity3D and LabVIEW. 
Next, LabVIEW is the software product in charge of actuating the crane mechanism. This product bundle was customized to acknowledge the information from MATLAB and afterward utilize this information to drive the mechanism continuously. The C-Rio 9012 controller is the gadget in charge of machine control in the crane mechanism. The controller was modified to listen to a pre-recognized port in the system at all times. At the point when the data sent by MATLAB through UDP has arrived at the port at the predefined time step, the C-Rio instantly grabs this information. The buoy position information is saved in the C-Rio memory to start with, and after that is used to compute and produce the right signal to the motor controller to track the motion produced by the magnetic rod. Since LabVIEW requires 20 data points of the buoy motion profile so as to figure the path that the machine will resemble, there is a slight wait between the moment when the data begins to arrive and the moment when the machine begins to move.

The Unity visual engine creates a game scene in Unity3D to display the interaction between the waves, the buoy and the mechanism. The game scene in the visual engine represents the intended working principle of the wave energy converter machine. That is, the absorbed wave energy is collected from the form of mechanical energy, which is then transformed into the electrical current used to power the LED light in this model. In this virtual reality (VR) environment, the buoy position and the wave motion generated by the MATLAB physics-based simulator are used to move the waves as well as the mechanism synchronously.

The input parameters to the MATLAB code are the size of the buoy and the wind speed and direction. These parameters will change the amount of the electricity generated to power the LED light as they will alter the interaction between the buoy and the waves. Furthermore, two sets of magnetic rods with different lengths are prepared, which can be replaced each other during the demonstration. These changes of parameters associated with buoy, waves and magnetic rods during demonstration will enhance students' engagement in the demonstration as well as their appreciation of the factors that are involved in the wave energy conversion process.

\section{PRE-DEMONSTRATION SET-UP}

The crane mechanism of the WEC machine is 50 " 12 " $\mathrm{x} 6$ " and weighted about $22 \mathrm{lbs}$. It consists of four main parts: the Crane Mechanism, the amplified LED Circuit, the Copper Solenoid and the Stepper Motor and Rail. Those are indicated in Figure 1 by numbers, (2), (4), (5) and (6), respectively. If there is no room in the transportation vehicle to hold the mechanism upright all the time, the movable parts of the mechanism needs to be dissembled for safety. Particularly, the stepper motor on the rail and the magnet along with copper coil should be dissembled before transportation. The first step of the disassembly is to remove the permanent magnet and the copper coil by taking out the bolt holding the L-shape bracket on which the coil is mounted. The second step is to remove the stepper motor by unscrewing the T-slot in the back of the rail. The LED circuitry can be left on the crane. The entire dissembling process takes about 5 minutes.

However, reassembling them back to operation will take a longer time in about 10 to 15 minutes. This is because the alignment between the permanent magnet, the copper coil and the spring has to be carefully adjusted to ensure the machine function properly.

\section{FORMAT OF PRESENTATION}

The presentation of the WEC system is performed to introduce the idea of renewable energy in the form of ocean waves to high school students. The total duration of the presentation is about 50 minutes to cover one class period. The WEC presentation has been conducted for a total of 65 students at two high schools: 38 students in the Camden County Technical High School in Sicklerville, New Jersey, and 27 in the Princess Anne High School in Virginia Beach, Virginia.

Each presentation is made of three parts: the power point lecturing, the hand-on demonstration, and the hardware assembly. The power-point presentation was made in the first 15 minutes of the class. The lecture introduced first the general topic of energy and the related issues that the society is currently facing. It is followed by the renewable and clean energy which led to the focus of the presentation, wave energy, its formation and collection. Final part of the presentation explained the working principle and three components of the WEC demonstration system. The former 
discussed the buoy motion driven by waves and converted into electricity through the change of magnetic flux. The major components of the WEC system include the physics simulation, virtual reality and hardware control.

Next is the hand-on section which lasts 20 - 30 minutes. This is the portion that includes both the demonstration and hands-on activities, where students can experience the WEC project and change the input parameters to enhance the concept introduced earlier. Students were given the chance to observe the MATLAB codes working with virtual reality code, Unity 3D for wave motion modeling, simulation and wave-buoy interaction codes. Additionally, they were challenged to examine the relationship between the wind speed and the amount of wave energy generated. The students were asked to adjust the wind speed and direction through GUI input to create a particular ocean wave that moves the buoy at different speed so as to generate different intensity in the light at the LED. Students, in this section, will be challenged to examine the connection between the wind speed and the intensity of the LED light as well. A higher wind speed will drive the buoy moved with larger amplitude as shown in the virtual ocean waves and lead to a brighter LED light.

Finally, when time was available, students were tasked to further explore their hands-on learning by reassembling the WEC system and changing the lengths of the springs and the magnets. The process would help the student to understand the factors that affect the energy harvesting efficiency by looking into the process of converting wave energy into electricity.

\section{SCHOOL VISITS}

A series of wave energy conversion presentations were made during the summer 2015 to students in two different high schools. One is the Camden County Technical School (CCTS) in Sicklerville, NJ and the other is the Princess Anne High School (PAHS) in Virginia Beach, VA (See Figures 3 and 4 for some snapshots of student activities).

CCTS is an inner city vocational school. According to Niche College, www.k12.niche.com, CCTS ranked $372^{\text {nd }}$ in the state of NJ, with a student body of 1,429. It is a diverse population with $35 \%$ white, $31 \%$ African American, $27 \%$ Hispanic, and $7 \%$ others. The average SAT score is 1420 , the math proficiency $52 \%$, and the reading proficiency $82 \%$. Among these $68.9 \%$ of students is eligible for free or reduced price lunch. In contrast, PAHS is an elite public school in Virginia Beach (ranked $15^{\text {th }}$ in the state of VA), constantly being the highest ranked high school in the city of Virginia Beach as well as the entire Hampton Roads region. Virginia Beach's only high school International Baccalaureate program is housed in PAHS, attracting gifted students from throughout the city. With a student body of 1,848 , the school's average SAT score is 1,800 , the math proficiency $67 \%$ and the reading proficiency $92 \%$. Among them $25.3 \%$ of students is eligible for free or reduced price lunch. The student population includes $57 \%$ white and $19 \%$ African American.

Two presentations were made to two different classes at CCTS; pre-engineering and chemistry. The total attendance is 38,37 of whom submitted the survey questionnaire after the presentation. The presentations were made to two APphysics classes at PAHS. 27 students participated and all turned in their survey questionnaire. The questionnaire asked students to answer a list of 4 questions. The survey questionnaire as listed in Table 1 gave the students a chance to voice their opinions on the working principle of the WEC (Question 1), the most interesting element of the project (Question 2), as well as the impact of the project on their science and engineering careers in the future (Questions 3 and 4).

In general, the WEC project presentation appeared to have a positive impact on students for two facets: introducing the new topic of renewable/wave energy, and encouraging their endeavor on future science/engineering career. The survey results are summarized in Diagram 1.

As a matter of fact, about $90 \%$ of 37 students in CCTS responded that the WEC project was very useful and $50 \%$ of that rated the project at the highest score of 5 in introducing the wave energy to them. While the virtual reality was singled out as the best part of the presentation with $40 \%$ votes, about the same amount of votes did confirm that all the components of the project are fun and helpful to them. Furthermore, more than $80 \%$ of them reported that the WEC project did a wonderful job in placing them on the side of liking science and had a good impact on encouraging them to pursue science and engineering careers. 
On the other hand, about $80 \%$ of 27 students in PAHS responded that the WEC project was very useful and $50 \%$ of that rates the project at the highest score of 5 in introducing the wave energy to them. The virtual reality was voted $30 \%$ as the best part of the presentation in comparison to $22 \%$ of the hardware platform, while $44 \%$ considered both made the project fun and helpful. Furthermore, more than $92 \%$ of them thought that the WEC project did a wonderful job making engineering and science attractive to them, though only $52 \%$ would like to pursue science or engineering careers.

Table 1. Survey Questionnaire

1. The presentation and the wave energy convertor help me to understand the process how electrical energy can be generated from wave motion.

( 1 being strongly disagree, 5 being strongly agree)

2. The presentation includes the power point, the virtual environment and the hardware platform. Among them, please select the part that is most interesting toyou.

(1: Power point presentation, 2: Virtual environment, 3: Hardware platform, 4: All of above)

3. After listening to the presentation and watching the wave energy conversion process, I do believe that it is fun and challenging to be an engineer or a scientist.

( 1 being strongly disagree, 5 being strongly agree)

4. After this presentation, I would more likely pursue a career in the future as an engineer or a scientist. ( 1 being strongly disagree, 5 being strongly agree)

Diagram 1. Survey Results at the Two Schools
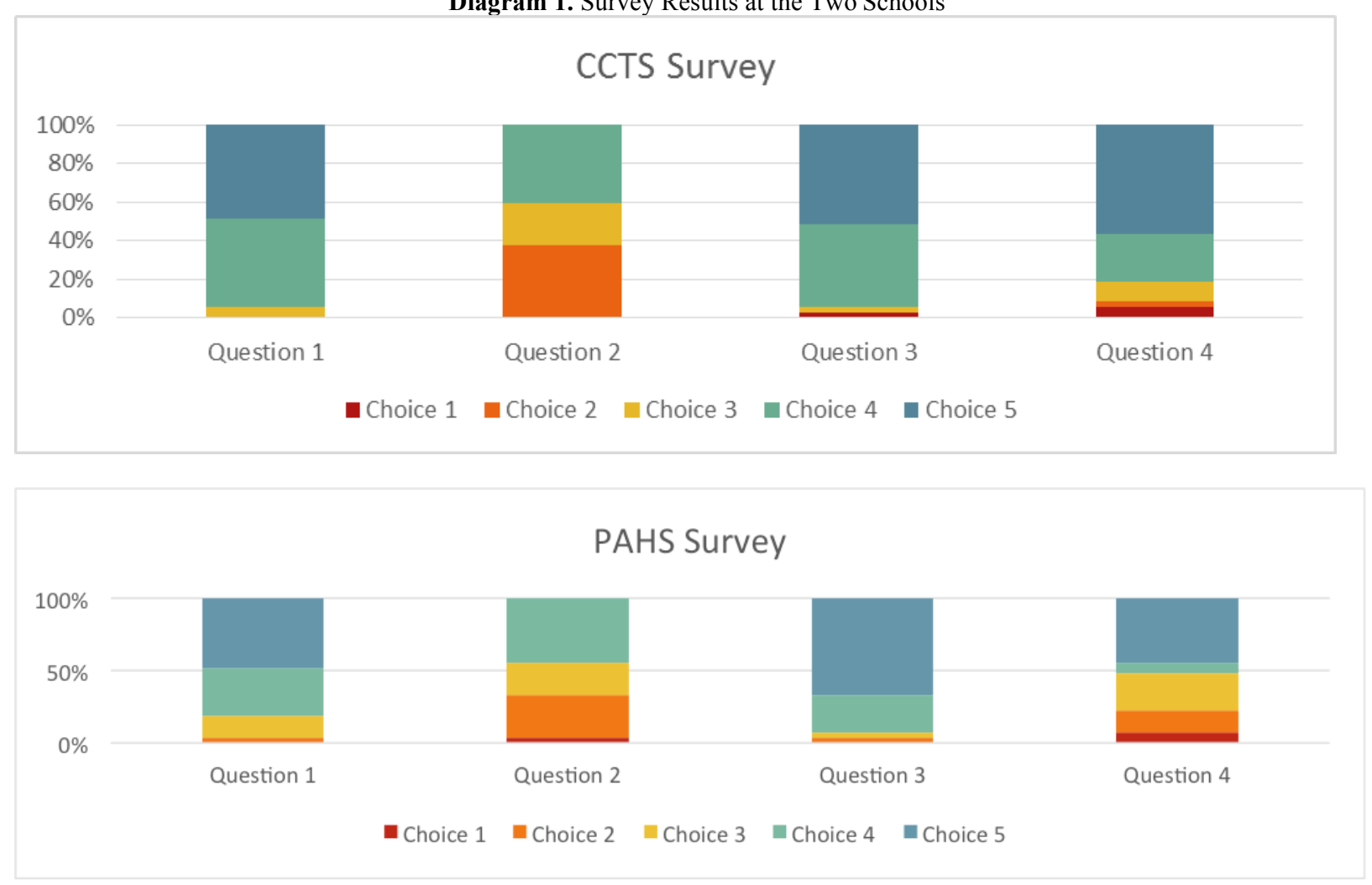
Figure 3. Students in PAHS change the buoy size

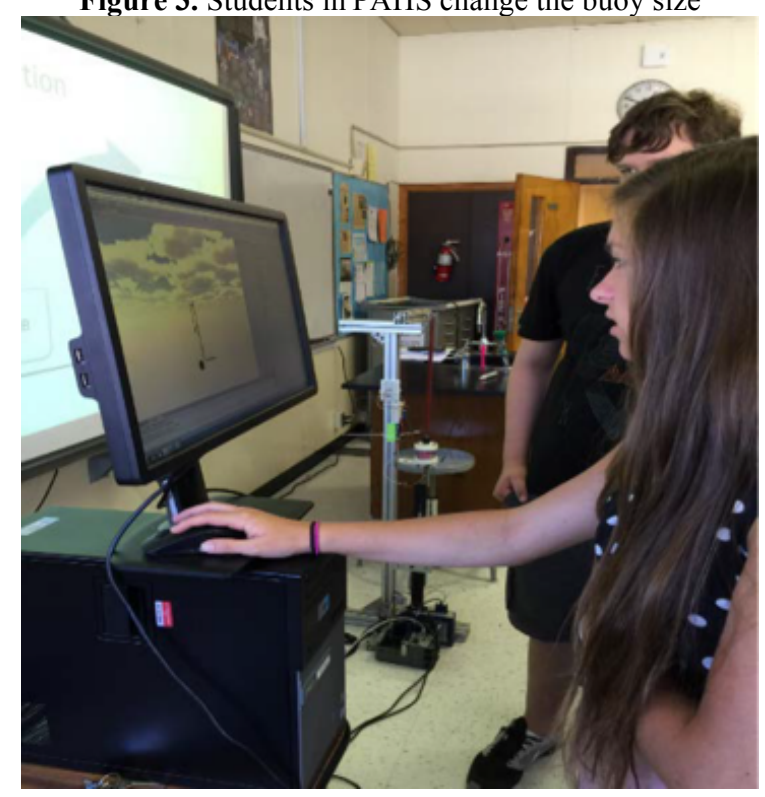

Figure 4. Students in CCTS change the magnetic rod

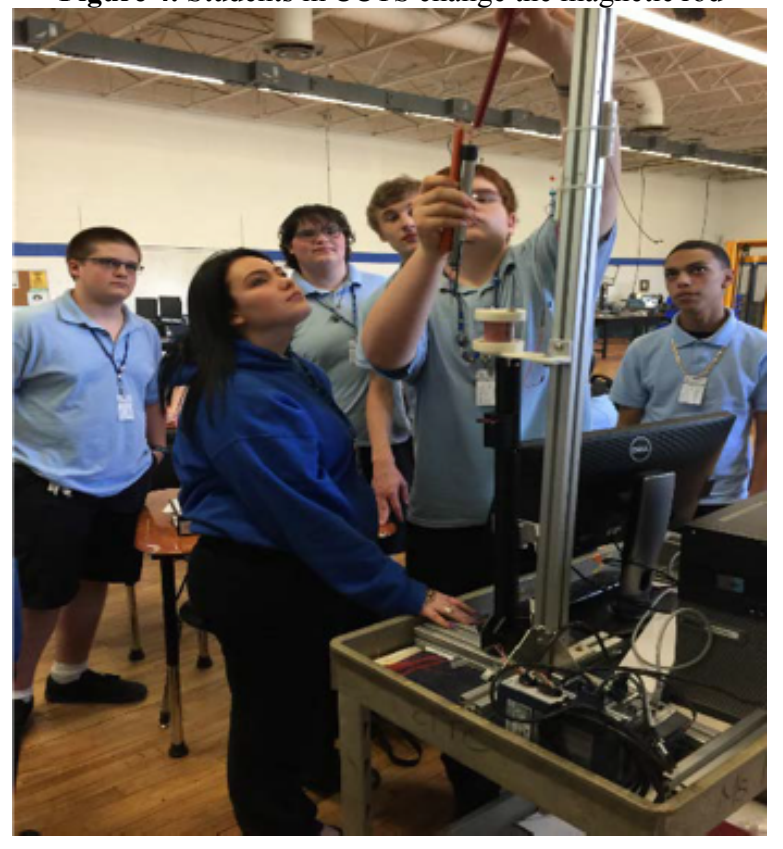

\section{CONCLUSIONS}

We have carried out an education project focused on wave energy and dedicated to the young generation. The project involved the set-up of a first-of-its-kind wave energy demonstration unit and the presentations to high school students at two different places. Surveys were conducted during each school visit, and the results indicated that the project objectives were satisfactorily met.

The novelty in the design of our wave energy convertor unit is the integration of hardware and software systems based on the concept of VR. Demonstrating wave energy using solely hardware devices is expensive; particularly, it requires 
a large wave tank and sufficient lab space to house the tank. As such, the scope of the demonstration is limited since students will have to come to the lab. On the other hand, using solely software system to demonstrate wave energy could be more affordable and portable, but short of being realistic, and the software demonstration would leave little for students' activities. Our hybrid demonstration system effectively combines the advantages, while overcoming the disadvantages, of the two. The designed unit is portable and transferrable, comes with minimum costs, and offers ample opportunities for the hands-on activities of students.

Based on this wave energy unit, our presentation to students combines lecturing, demonstration, and hand-on learning, with a goal of actively and effectively communicating the concept of renewable energy to students and promote their interest in pursuing a career in science and engineering. The lecture and demonstration parts are focused on the basic knowledge, whereas the hands-on learning activities aim to engage students for better understanding of the wave energy concept and its technological application.

Our wave energy presentations at two public schools, Camden County Technical School in New Jersey, and Princess Anne High School in Virginia, have both been successful. The survey results from students were dominantly positive in both the contents and impacts of the presentation. In particular, students were mostly interested in the new knowledge introduced to them, and were motivated for a career in related science and engineering fields. Most students showed strong interest in the wave energy demonstration unit, especially the virtual reality component. Building on these pilot project experiences, our future plan is to not only conduct the physical presentations at more places, but also to communicate and share the project details through internet and digital means (using interactive web-based simulation platform, recorded videos, etc.), to significantly enlarge the size of possible audience and maximize the impacts of the education project. We also hope to collect more data to help with the statistical assessment of the project outcome.

\section{ACKNOWLEDGMENTS}

This work was partially supported by the National Science Foundation under Grant Number 1245769, and by the Tennessee Higher Education Commission through a CEACSE grant. The authors are grateful to the Editor and the two anonymous reviewers for their helpful comments to improve this paper.

\section{AUTHOR BIOGRAPHY}

Trongnghia Huynh is a graduate student in the Department of Mechanical and Aerospace Engineering at Old Dominion University.

Gene Hou is a Professor of Mechanical and Aerospace Engineering at Old Dominion University.

Jin Wang is a Professor of Applied Mathematics at the University of Tennessee, Chattanooga.

\section{REFERENCES}

Falnes, J. (2002). Ocean Waves and oscillating systems. New York: Cambridge University Press.

Cruz, J. (2010). Ocean Wave Energy. Heidelberg, Germany: Springer.

McCormick, M.E. (2007). Ocean Wave Energy Conversion. New York: Dover Publications.

Westwood, P.S. (2008). What Teachers Need to Know about Teaching Methods. Victoria, Australia: ACER Press.

Renkl, A., Atkinson, R.K., Maier, U.H., \& Staley, R. (2002). From example study to problem solving: Smooth transitions help learning. Journal of Experimental Education, 70 (4), 293-315.

Kyriacou, C. (1992). Active Learning in Secondary School Mathematics. British Educational Research Journal, 18 (3), $309-318$.

Hearns, M.K., Miller, B.K., \& Nelson, D.L. (2010). Hands-on learning versus learning by demonstration at three recall points in university students. Occupation, Participation and Health, 30, 169-171.

Kontra, C., Lyons, D.J., Fischer, S.M., \& Beilock. S.L. (2015). Physical experiences enhance science learning. Psychological Science, 26, 737-749.

Hillman, C.N. (2011). The effects of hands-on learning versus learning by demonstration on memory in community dwelling older adults. University of Toledo Master's and Doctoral Projects, Paper 192. 
Beard, C. (2010). The Experiential Learning Toolkit: Blending Practice with Concepts. London, UK: Kogan Page.

Itin, C.M. (1999). Reasserting the Philosophy of Experiential Education as a Vehicle forChange in the 21st Century. The Journal of Physical Education, 22(2), 91-98.

Huynh, T., Hou, G., Wang, J., Hou, M., \& Kotinis, M. (2015). A hybrid virtual reality simulation system for wave energy conversion. International Journal of Computational Engineering Research, 5(6), 50-60.

Mikropoulos, T.A., \& Natsis, A. (2011). Educational Virtual Environments: A Ten-Year Review of Empirical Research (19992009). Computers \& Education, 56, 769-780.

Gadre, A., Cudney, E., \& Corns, S. (2011). Model Development of a Virtual Learning Environment to Enhance Lean Education. Procedia - Computer Science, 6, 100-105.

Ong, S.K., \& Mannan, M.A. (2004). Virtual Reality Simulations and Animations in a Web-based Interactive Manufacturing Engineering Module. Computers \& Education, 43,361-382.

Dalgarno, B., Bishop, A.G., Adlong, W., \& Bedgood Jr., D.R. (2009). Effectiveness of a Virtual Laboratory as a Preparatory Resource for Distance Education Chemistry Students. Computers \& Education, 53, 853-865.

Saleh, K.F., Mohamed, A.M., \& Madkour, H. (2009). Developing Virtual Laboratories Environments for Engineering Education. International Journal of Arts and Sciences, 3(1), 9-17.

Goeser, P.T., Johnson, W.M., Hamza-Lup, F.G., \& Schaefer, D. (2011). VIEW - A Virtual Interactive Web-based Learning Environment for Engineering. IEEE Advances in Engineering Education, 2(3), 24.

Hu, X., \& Zeigler, B.P. (2005). Model Continuity in the Design of Dynamic Distributed Real- Time Systems. IEEE Transactions on Systems, Man and Cybernetics - Part A: Systems and Humans, 35(6), 867-878.

Richardson, J., Adamo-Villani, N., Carpenter, E., \& Moore, G. (2006). Designing and Implementing a Virtual 3D Mircrocontroller Laboratory Environment. In: Proceedings of the36th ASEE/IEEE Frontiers in Education Conference, October 28-31, San Diego, CA.

Morris, D., Sewell, C., Blevins, N., Barbagli, F., \& Salisbury, K. (2004). A Collaborative Virtual Environment for the Simulation of Temporal Bone Surgery. Medical Image Computing and Computer-Assisted Intervention, Lecture Notes in Computer Science, 3217,319-327.

Fu, Y., Chen, C., \& Cai, J. (2011). Research on Virtual Reality Simulation System of Safe Navigation Environment in Three Gorges Reservoir Area. Procedia - Environmental Science, 10, 331-336.

Crosier, J.K., Cobb, S., \& Wilson, J.R. (2002). Key Lessons for the Design and Integration of Virtual Environments in Secondary Science. Computers \& Education, 38, 77-94. 
NOTES 